\title{
Peer Reviewers for the Journal of the American Board of Family Medicine in 2018
}

\author{
Marjorie A. Bowman, MD, MPA, Anne Victoria Neale, PhD, MPH, \\ Dean Seehusen, MD, MPH, and Phil Lupo, MLIS (J Am Board Fam Med 2019;32: \\ 126-129.)
}

To our peer reviewers: We thank you for your time, expertise, and dedication to the fournal of the American Board of Family Medicine ( $7 A B F M)$.

$7 A B F M$ peer reviewers serve a vital role in contributing to the field of family medicine and to its scientific community. We take this opportunity to acknowledge and thank our peer reviewers for their support during the past year. In 2018, 372 individuals provided 461 peer reviews and advice regarding the suitability of articles for publication in the FABFM. Reviewers took an average of 18 days to submit a review.

\section{Top Reviewers}

Each of the manuscripts submitted to the $7 A B F M$ is first reviewed by the editors and, if deemed suitable

Conflict of interest: The authors are editors of the $7 A B F M$. for peer review, is evaluated and scored by volunteer reviewers. In the list below, peer reviewers with an asterisk next to their name are our top reviewers for 2018 based on evaluation scores and the quantity of critiques provided.

\section{Seeking New Reviewers}

We also enjoy welcoming new peer reviewers into the fold. Peer review is a vital service that helps authors and researchers improve their work. Reviewers are always free to decline a request or to indicate periods of unavailability. If you would like to volunteer to review for the $7 A B F M$, please download and complete the Peer Reviewer Form, which can be accessed online at https://www.jabfm. org/content/information-reviewers. Then, e-mail it, along with your curriculum vitae, to jabfm@med.wayne.edu, or fax it to 313-577-6718. Thank you!

\begin{tabular}{lll}
\hline 2018 Peer Reviewers & & \\
Adrienne Ables* & Sonal Admane & Krishna Agarwal \\
David Agerter & Kathryn Aikin & Katherine Akers \\
Steven Albert & Sommer Aldulaimi* & Gwen Alexander \\
Grace Alfonsi & Deborah Allen & Kurt Angstman \\
Ann Gene Anthony & Sarah Arias* & Gary Asher \\
Thomas Atkinson & Russell Attridge & Elisabeth Backer \\
Bijal Balasubramanian & Matthew Barnes & Donald Barr* \\
Tyler Barreto & Kirsten Barrett & Mary Barron \\
Gillian Bartlett & Ian Bennett* & George Bergus \\
Juliann Binienda* & Helen Binns & Rajshekhar Bipeta \\
Richard Birtwhistle & Arthur Blain & Alan Blum \\
Debra Boardley & George Bock & John Boltri \\
Amanda Borsky & Pranjal Boruah & Matthew Breeden \\
Stephanie Brennhofer & Jay Brieler & Shandra Brown Levey \\
Christopher Bunt & Lucinda Buys & Elena Byhoff* \\
Leigh Callahan & Mollie Canzona* & Timothy Carey \\
Beth Careyva & Caroline Carlin & Donald Chaffee \\
\hline
\end{tabular}




\begin{tabular}{|c|c|c|}
\hline Candice Chen & Frederick Chen* & Saleem Chowdhry \\
\hline Carolyn Chu & Charles Clinch & Kenneth Cohen \\
\hline Erika Cottrell* & Anastasia Coutinho & Benjamin Crabtree \\
\hline Peter Crampton & Monica Crane & Steven Crane \\
\hline Paul Crawford & Timothy Crawford* & Peter Croft \\
\hline Michael Crouch & Brian Crownover* & Larry Culpepper \\
\hline Amy Cunningham & Joshua Cusick-Lewis & Peter Danis \\
\hline Jaividhya Dasarathy* & Alan David & Joshua Davis \\
\hline Melinda Davis & Dawn Davis & Philip Day \\
\hline Peter Del Fante & Vanessa Diaz & John Dorsch \\
\hline Steven Dosh & Chyke Doubeni* & Marguerite Duane \\
\hline Aimee Eden & Milton Eder & Samuel Edwards \\
\hline Ellen Einterz & William Elder & Nancy Elder* \\
\hline John Ely & John Epling & Philip Eskew \\
\hline Anna Espinoza & Krista Farey & Anum Fasih \\
\hline Greg Feero & Richard Fehring & Steven Feldman \\
\hline Joshua Fenton* & Douglas Fernald & Jeanne Ferrante* \\
\hline Edgar Figueroa* & Kenneth Fink* & Kevin Fiscella* \\
\hline Gemmae Fix & Susie Fletcher & Jonathan Foley \\
\hline Elliot Frank & Gary Franklin & Adam Franks \\
\hline William Freeman & Joshua Freeman & Kimberly Fulda* \\
\hline Heidi Gaddey & Anne Gaglioti* & Sim Galazka* \\
\hline Iesha Galloway-Gilliam & Jinal Gangar & David Garcia \\
\hline Nicole Gastala & Dan Gelfman & Cynthia Geppert \\
\hline Abhijeet Ghatol & Jayashri Ghosh* & Valerie Gilchrist \\
\hline Mary Lou Gillard & Julie Gleason-Comstock & Marji Gold \\
\hline Stephanie Gold & Rachel Gold* & Rise Goldstein \\
\hline Ralph Gonzales & Cesar Gonzalez & Rachel Gorwitz \\
\hline John Gossey* & Joseph Gravel & Beverly Green* \\
\hline Larry Green* & Jeffrey Greenwald & Kim Griswold \\
\hline Alexander Guirguis & Amber Gum & David Hahn* \\
\hline Jacqueline Halladay & Allan Halpern & Kathryn Harmes \\
\hline Ila Harris & Scott Hartman & Robert Hatch \\
\hline Clare Hawkins & Diana Heiman & Robin Helm \\
\hline Margaret Helton & Sarah Hemeida & Jennifer Hemler \\
\hline Vincent Hevern & John Hickner* & Neil Holland \\
\hline Richard Holloway & John Holmes & Jennifer Horn \\
\hline Rivka Horowitz & Michael Horst & Susan Hsieh \\
\hline William Hueston & Miranda Huffman & Nathalie Huguet \\
\hline Steinar Hunskaar & Katherine Hurst & David Hyman \\
\hline Jihad Irani & Jamal Islam & Henry Ivey \\
\hline Muhammad Javed & Anthony Jerant & Masahito Jimbo \\
\hline Pamela Jo Johnson & Mark Johnson* & David Kealy \\
\hline Rick Kellerman & Allison Kempe & Rodger Kessler \\
\hline Morteza Khodaee & Autumn Kieber-Emmons & Kim Kimminau* \\
\hline Dana King & N Kolb & Kelly Koren \\
\hline Kurt Kroenke & Robin Kruse & Irina Kudrina \\
\hline Abhishek Kulkarni & Erine Kupetsky & Anton Kuzel* \\
\hline Maude Laberge & William Lambert & Paul Lavender \\
\hline Aaron Lear & Patricia Lenahan & Robert Lennon \\
\hline
\end{tabular}




\begin{tabular}{|c|c|c|}
\hline Joanne Leovy & Brian Lerner & Lenard Lesser \\
\hline Barcey Levy & Winston Liaw* & Clare Liddy \\
\hline Deerajnath Lingutla* & Paula Darby Lipman & Benjamin Littenberg \\
\hline Sean Lucan* & Thea Luig & Hillary Lum \\
\hline Diane Madlon-Kay & Michael Magill* & Martin Mahoney \\
\hline Rose Maly & David Mannino & Jo-Anne Manski-Nankervis \\
\hline Katherine Margo & Jason Marker* & Tsveti Markova \\
\hline Cara Marshall & Eric Mast & Ann McAlearney \\
\hline Adelaide McClintock & Jane McElroy & Ivan Mefford \\
\hline Taverna Melanie & Michelle Mengeling & Maria Mironidou-Tzouveleki \\
\hline Kei Miyazaki & Jennifer Molokwu & Gerardo Moreno \\
\hline Mark Morgan & Christopher Morley* & Megan Morris \\
\hline Anne Mounsey & Karen Muchowski & John Muench \\
\hline Zsolt Nagykaldi* & Mohammad Obadah Nakawah & Raj Narayan \\
\hline Susan Nash & Isaac Navarro & Warren Newton \\
\hline Kari Nilsen & Thomas Norris & Thomas Northrup \\
\hline Molly Nowels* & Alane O'Connor & Robert O’Connor \\
\hline Jason O’Grady & David O’Gurek & Thomas Oates \\
\hline Robert Oh & Kristine Olson & Darrell Over \\
\hline Janet Page & Joseph Palamar & Michael Parchman \\
\hline Donald Pathman* & Michael Peabody & William Pearson \\
\hline Randolph Pearson & Brian Penti* & Allen Perkins \\
\hline Lars Peterson & Stephen Petrany & William Phillips \\
\hline Margaret Planta & Michael Potter* & Perry Pugno \\
\hline Elizabeth Quigley & Howard Rabinowitz & T Raju \\
\hline Meghan Raleigh & Stephen Ratcliffe & Brian Reamy \\
\hline James Reschovsky & Peter Reynolds & Robert Rhyne \\
\hline Martha Riddell & Deborah Rinehart & Jill Rissi \\
\hline Peter Rives & Pamela Rockwell & Rene Rodriguez-Gutierrez \\
\hline Thomas Rosenthal & Robert Ross & Walter Rosser \\
\hline Mack Ruffin* & Terry Ruhl & George Rust \\
\hline Saria Saccocio & Carolyn Sachs & Amelia Sattler \\
\hline Margot Savoy & Greg Sawin & Joseph Scherger \\
\hline Jeffrey Schievenin & Sarah Scholle & Sarina Schrager* \\
\hline Miriam Schwartz & Kendra Schwartz* & Thomas Schwenk \\
\hline Peter Selwyn & David Serlin & Jessica Servey \\
\hline Patricia Sexton* & Fadi Shamoun & Michelle Sherman \\
\hline Sasha Shillcutt & Debbie Ann Shirley & William Sieber* \\
\hline Aditya Simha & Anne Simpson & Vijay Singh \\
\hline Gurmukh Singh & Peter Smith & Samantha Solimeo \\
\hline Lori Solomon & John Standridge & Elizabeth Staton \\
\hline Michael Stehney & Rosemary Stevens & Earl Stewart \\
\hline Melissa Stiles & Glen Stream & George Strelioff \\
\hline Walton Sumner & John Sutherland & Paul Swank \\
\hline Divya Talwar* & Daniel Tancredi & Hazel Tapp* \\
\hline Yhenneko Taylor & Chris Terpening & Tom Thacher \\
\hline Manish Tiwari & Barbara Tobias & Sebastian Tong \\
\hline Daniel Van Durme & Constance van Eeghen & David Vearrier \\
\hline Bonnie Vest* & Kirsten Vitrikas & Arnold Wald \\
\hline Eric Wall* & Melissa Walls & Gregg Warshaw \\
\hline Katherine Watkins & Sally Weaver & Stephanie Weiner \\
\hline David Weldy & Andrea Wendling & Andrea Wessell \\
\hline John Westfall & Karen Whalen* & John White \\
\hline
\end{tabular}




\begin{tabular}{lll}
\hline Brett White & Robert Williams & Calvin Wilson \\
Robin Winter & Paula Witt-Enderby & Jason Womack \\
W Woods & Tsu-Yin Wu & Imam Xierali* \\
Joseph Yancey & Richard Young* & Jonathan Yun \\
\hline
\end{tabular}

*Top reviewers in 2018 (based on quantity and quality of peer reviews written).

To see this article online, please go to: http://jabfm.org/content/32/2/126.full. 